\title{
DESEMPENHO DE MEMBRANAS POLIMÉRICAS COMERCIAIS DE NANOFILTRAÇÃO E OSMOSE INVERSA NA SEPARAÇÃO E FRACIONAMENTO DE ÓLEO DE MACAÚBA UTILIZANDO $\mathrm{CO}_{2}$ DENSO
}

\author{
K. REZZADORI ${ }^{1}$, J. C. M. SILVEIRA ${ }^{1}$, J. G. VENERAL ${ }^{1}$, F. M. PENHA ${ }^{1}$, M. DI LUCCIO ${ }^{1}$, J. C. C. \\ PETRUS ${ }^{1}$ \\ ${ }^{1}$ Universidade Federal de Santa Catarina, Departamento de Engenharia Química e Engenharia de \\ Alimentos \\ E-mail para contato: jpetrus@enq.ufsc.br
}

\begin{abstract}
RESUMO - O presente estudo teve como objetivo investigar o desempenho de uma membrana de nanofiltração (NP030) e de uma membrana de osmose inversa (ORAK) na permeação e retenção de uma mistura de óleo de macaúba e $\mathrm{CO}_{2}$ sub ou supercrítico. Foram utilizadas condições sub $\left(80 \mathrm{bar} / 20^{\circ} \mathrm{C}\right)$ e supercríticas $\left(130 \mathrm{bar} / 40{ }^{\circ} \mathrm{C}\right)$ em sistema contínuo na concentração de $50 \%$ de óleo na mistura. $\mathrm{O}$ desempenho das membranas foi avaliado em função do fluxo mássico e do índice de retenção do óleo (\%); e ácidos graxos. Observou-se uma redução do fluxo permeado de óleo (cerca de 2 x) ao longo do processo para a condição supercrítica. O índice de retenção de óleo foi de $85 \%$ e $95 \%$ para as membranas NP030 e ORAK, respectivamente, também em condições supercríticas. Com relação à composição dos óleos em termos de ácidos graxos, foi observada maior retenção para o ácido palmitoleico (23 \%), com a membrana ORAK, enquanto que a membrana NP030 apresentou maior retenção para os ácidos mirístico (12 \%) e caprílico (12\%). Em condições subcríticas não foi observada permeação de óleo, comprovando o maior poder de solvatação do $\mathrm{CO}_{2}$ supercrítico. Com base nestes resultados verificou-se o potencial de uso de membranas em sistemas supercríticos para recuperação do solvente sem necessidade de descompressão do sistema.
\end{abstract}

\section{INTRODUÇÃO}

Nos últimos anos a indústria química e de alimentos tem buscado formas de desenvolver produtos de melhor qualidade, para diminuir o consumo energético e valorizar os subprodutos gerados. Alguns processos de fracionamento e concentração se tornaram clássicos na literatura por serem estudados há muitos anos, atingindo um grau de maturidade tecnológica, enquanto processos alternativos, como os processos de separação por membranas (PSM) são ainda recentes e cujas possibilidades de otimização e aplicação ainda estão sendo aprimoradas (Pokrywiecki, 2006).

$\mathrm{Na}$ indústria de alimentos destaca-se o estudo e utilização de processos de separação com membranas de osmose inversa (OI) e nanofiltração (NF) em sistemas aquosos, como na concentração de compostos funcionais (Benedetti et al., 2011) de proteínas do leite e do soro 
(Debon et al., 2010) e também na clarificação de sucos (Rezzadori et al., 2013). Nas últimas décadas vem se destacando também a utilização dessas membranas em sistemas não aquosos, como na desacidificação e clarificação de óleos vegetais (Araki et al., 2010; Tres et al., 2009) e na separação de compostos naturais de óleos essenciais (Carlson et al., 2005; Martínez et al., 2012).

Em paralelo, diversos processos que utilizam como solvente o dióxido de carbono denso vêm sendo desenvolvidos. $\mathrm{O} \mathrm{CO}_{2}$ tem a vantagem de ser atóxico e inerte, além de, como solvente, ter a possibilidade de ajuste da sua seletividade e do seu poder de solubilização pelo ajuste da temperatura e da pressão empregadas no processo (Pokrywiecki, 2006).

$\mathrm{O}$ uso de processos com membranas simultâneos a processos com $\mathrm{CO}_{2}$ denso pode apresentar vantagens adicionais, como evitar etapas de despressurização e aperfeiçoar a separação de compostos pelo efeito conjunto de seletividade do $\mathrm{CO}_{2}$ e da membrana (Akin e Temelli, 2012 $\left.2^{\mathrm{a}, \mathrm{b}}\right)$. Além disso, de acordo com Araki et al. (2010), a presença de solventes orgânicos ou do $\mathrm{CO}_{2}$ pode levar a maiores fluxos de permeado e, portanto, aumentar a viabilidade destas operações. No entanto, o desenvolvimento dessa alternativa tecnológica está vinculado à estabilidade química e a resistência mecânica das membranas utilizadas.

Umas das formas de se avaliar a estabilidade e o desempenho das membranas em sistemas não aquosos é a caracterização funcional. Parâmetros funcionais consistem na exploração de sua permeabilidade em relação a determinados solventes orgânicos, além da determinação dos coeficientes de retenção e fatores de separação em misturas soluto/solvente orgânico (CHERYAN, 2005).

Neste contexto, o objetivo desse trabalho foi investigar o desempenho de membranas poliméricas comerciais de nanofiltração (NF) e osmose inversa (OI) na permeação e retenção de óleo de macaúba em condições sub e supercríticas.

\section{MATERIAL E MÉTODOS}

Duas membranas comerciais foram estudadas, sendo que uma de NF e outra de OI. As principais características de cada membrana, de acordo com informações dos fabricantes, estão descritas na Tabela 1.

O óleo bruto da amêndoa da macaúba, obtido por extração mecânica, foi adquirido da empresa Cocal Óleos Especiais Ltda (Abaeté, Minas Gerais). Assim que recebido, o óleo foi acondicionado em embalagens plásticas opacas de $1 \mathrm{~L}$, com atmosfera inerte $\left(\mathrm{N}_{2}\right)$ e congelados em freezer (Eletrolux Freezer/Cooler H500) a aproximadamente $-18{ }^{\circ} \mathrm{C}$, a fim de minimizar degradações. A degomagem do óleo foi realizada pelos métodos propostos por Fett e Moretto (1998) e De e Patel (2010), com adaptações. 
O diagrama do sistema de permeação com solvente supercrítico está representado esquematicamente no diagrama da Figura 1.

Tabela 1 - Características das membranas que foram utilizadas nos ensaios

\begin{tabular}{lllccccc}
\hline Membrana & Fabricante & Material & $\begin{array}{c}\text { MMC }^{\mathrm{d}} \\
(\mathrm{Da})\end{array}$ & $\begin{array}{c}\text { P máx } \\
(\mathrm{bar})\end{array}$ & $\begin{array}{c}\text { T máx } \\
\left({ }^{\circ} \mathrm{C}\right)\end{array}$ & $\begin{array}{c}\text { Faixa } \\
\text { de } \mathrm{pH}\end{array}$ & $\begin{array}{c}\text { Rejeição } \\
(\%)\end{array}$ \\
\hline NP030 & Microdyn & Polietersulfona & 400 & 40 & 95 & $0-14$ & $80-97^{\mathrm{a}}$ \\
OR AK & Osmonics & Poli(amida) TFC & - & 27 & 50 & $4-11$ & $99^{\mathrm{b}}$ \\
\hline
\end{tabular}

a Rejeição em $\mathrm{Na}_{2} \mathrm{SO}_{4}\left(20^{\circ} \mathrm{C} ; 40\right.$ bar $)$

b Rejeição em $\mathrm{NaCl}\left(25^{\circ} \mathrm{C} ; 15.5\right.$ bar $)$

c TFC: Thin film composite

${ }^{\mathrm{d}} \mathrm{MMC}$ : Massa molar de corte

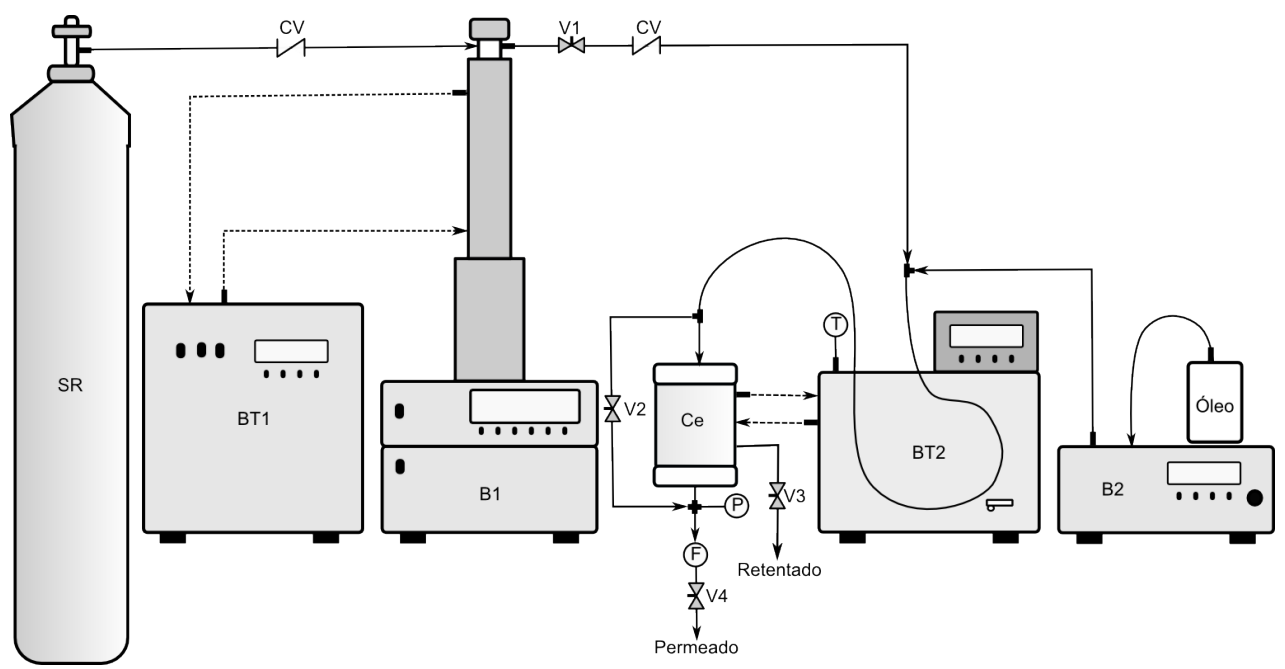

Figura 1 - Unidade de filtração associado a $\mathrm{CO}_{2}$ denso e óleo de macaúba em sistema contínuo: cilindro de $\mathrm{CO}_{2}$ (SR); célula de filtração (Ce); bomba seringa de alta pressão (B1); bomba HPLC (B2); válvula de uma via (CV); válvula micrométrica (V1); válvula esfera on/off (V2); válvula agulha micrométrica (V3 e V4); transdutor de pressão (P); fluxímetro (F); banho termostático (BT1 e BT2); termopar (T).

A válvula do cilindro SR foi aberta e câmara interna da bomba B1 (ISCO, modelo D260, Teledyne ISCO Inc., Lincon, NE) foi abastecida até o volume máximo de $1000 \mathrm{~mL}$ de $\mathrm{CO}_{2}$ à pressão do cilindro. A válvula micrométrica $\mathrm{V} 1$ neste momento permanece fechada, impedindo a passagem de $\mathrm{CO}_{2}$ para o sistema. 


\section{9 a 22 de outubro de 2014 \\ Florianópolis/SC}

A membrana foi colocada na célula de permeação Ce sobre uma placa perfurada de aço inox de diâmetro $52 \mathrm{~mm}$ e espessura $5 \mathrm{~mm}$. A área efetiva de membrana foi de $2,624.10^{-3} \mathrm{~m}^{2} \mathrm{e} o$ regime de escoamento utilizado foi o perpendicular. A célula Ce também é encamisada e sua temperatura foi controlada pelo banho termostático BT2 (Tecnal, modelo TE-2005, Brasil).

Antes de serem dispostas na célula $\mathrm{Ce}$, as membranas foram recortadas em formato circular com diâmetro aproximado de $50 \mathrm{~mm}$ e ficaram submersas em etanol por duas horas. A célula $\mathrm{Ce}$ foi fechada e acoplada ao sistema. As válvulas micrométricas (Hoke, modelo 1315G4Y, EUA) $\mathrm{V}_{3}$ e V4 foram fechadas completamente, enquanto a válvula V2 permaneceu aberta para possibilitar o sistema chegar à pressão desejada.

A válvula $V_{1}$ foi aberta lentamente, a fim de evitar danos na estrutura da membrana pela elevação abrupta da pressão da célula, que foi monitorada pelo transdutor de pressão P. A pressão do sistema foi elevada de $10 \mathrm{em} 10$ bar pelo controlador da bomba B1 até atingir a pressão desejada.

A válvula on/off V2 (Swagelok, modelo SS-83KS) foi fechada para possibilitar a variação da pressão transmembrana. O fluxo de $\mathrm{CO}_{2}$ no sistema foi controlado pela bomba $\mathrm{B} 1$ para $3 \mathrm{~mL} \cdot \mathrm{min}^{-1}$, enquanto o fluxo de óleo de macaúba foi controlado pela bomba B2 (HPLC, Digital Série III, Acuflow, USA) para $3 \mathrm{~mL} \cdot \mathrm{min}^{-1}$. As duas correntes se encontram em uma conexão T e percorrem 1 metro de tubulação de aço inoxidável submerso no banho termostático BT2 (Tecnal, modelo TE-2005, Brasil), afim de possibilitar a mistura de óleo e solvente entrar em equilíbrio antes do contato com a membrana.

A válvula V3 foi usada para controlar a pressão do sistema anterior à membrana, ou seja, a pressão da bomba B1 para 80 ou 130 bar, de acordo com a condição utilizada. A válvula V4 foi usada para controlar a pressão transmembrana $(\Delta \mathrm{P})$ desejada, que nos ensaios realizados foi de 50 bar, sendo esta aferida pelo transdutor P (Novus, modelo HUBA-511, Suíça). Na Tabela 2 estão apresentadas as condições de pressão, temperatura e $\Delta \mathrm{P}$ utilizadas nos ensaios. Todos os ensaios foram realizados em duplicata.

Tabela 2 - Condições de pressão, temperatura e $\Delta \mathrm{P}$ utilizadas nos ensaios de permeação de óleo de macaúba e $\mathrm{CO}_{2}$ denso

\begin{tabular}{cccccc}
\hline Ensaio & Membrana & Temperatura $\left({ }^{\circ} \mathrm{C}\right)$ & Pressão B1 (bar) & Pressão P (bar) & $\Delta \mathrm{P}($ bar $)$ \\
\hline 1 & NP030 & 20 & 80 & 30 & 50 \\
2 & NP030 & 40 & 130 & 80 & 50 \\
3 & OR AK & 20 & 80 & 30 & 50 \\
4 & OR AK & 40 & 130 & 80 & 50 \\
\hline
\end{tabular}

As condições de operação foram escolhidas com base em experimentos prévios. Amostras de permeado foram coletadas em frascos de vidro âmbar, previamente pesados, de 5 em 5 minutos. O tempo total de coleta foi de $60 \mathrm{~min}$, contados a partir da primeira gota de óleo do permeado. Os frascos foram pesados após a coleta para o calculo do fluxo mássico. 
A composição química das frações de permeado e retido foi determinada por cromatografia gasosa acoplada a um detector seletivo de massa (GC-MS) (Shimadzu, modelo GCQT8030, USA), com um software GC-MS (versão 4.11) usando as seguintes condições: coluna Rtx-WAX $25 \mathrm{~m}$ x 0,25 mm x 0,25 um, injeção split 1:50, temperatura do injetor $250{ }^{\circ} \mathrm{C}$, temperatura da fonte de ionização $250{ }^{\circ} \mathrm{C}$, temperatura da interface $250{ }^{\circ} \mathrm{C}$ e velocidade de escoamento do gás hélio de $1,8 \mathrm{~mL} \cdot \mathrm{min}^{-1}$.

Os índices de retenção foram determinados pela Equação 1, conforme Sarmento (2007).

$$
I R=\left(1-\frac{C p}{C r}\right) \times 100
$$

sendo: $\mathrm{IR}=$ índice de retenção $(\%)$

$\mathrm{Cp}=$ concentração de ácidos graxos no permeado

$\mathrm{Cr}=$ concentração de ácidos graxos no retentado

\section{RESULTADOS E DISCUSSÃO}

Para as membranas NP030 e ORAK foi observada permeação do óleo somente na condição supercrítica. Isso pode ser devido ao menor poder de solvatação do $\mathrm{CO}_{2}$ na condição subcrítica. Em condições subcríticas, a viscosidade dos líquidos comprimidos é maior que nos fluidos supercríticos, o que provavelmente é a razão para não ter sido observada permeação.

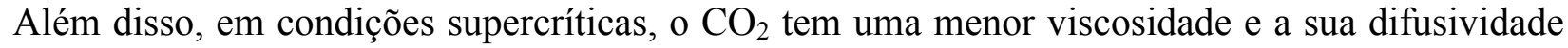
pode ser duplicada.

Conforme pode ser observado na Figura 2, o comportamento do fluxo de óleo através das membranas com o tempo é típico, ou seja, há um decrescimento acelerado do fluxo em um primeiro momento, seguido de uma tendência à estabilização deste. O fluxo de óleo de macaúba sofre uma redução de aproximadamente 2 vezes durante os primeiros 25 min de ensaio e tende a um valor estável, para as duas membranas. Essa redução se deve, provavelmente, à compactação da membrana ou ao fenômeno da polarização por concentração e ao desenvolvimento de uma camada de óleo na superfície da membrana, que implica na alteração da resistência da membrana durante o processo de permeação (MULDER, 2000). 

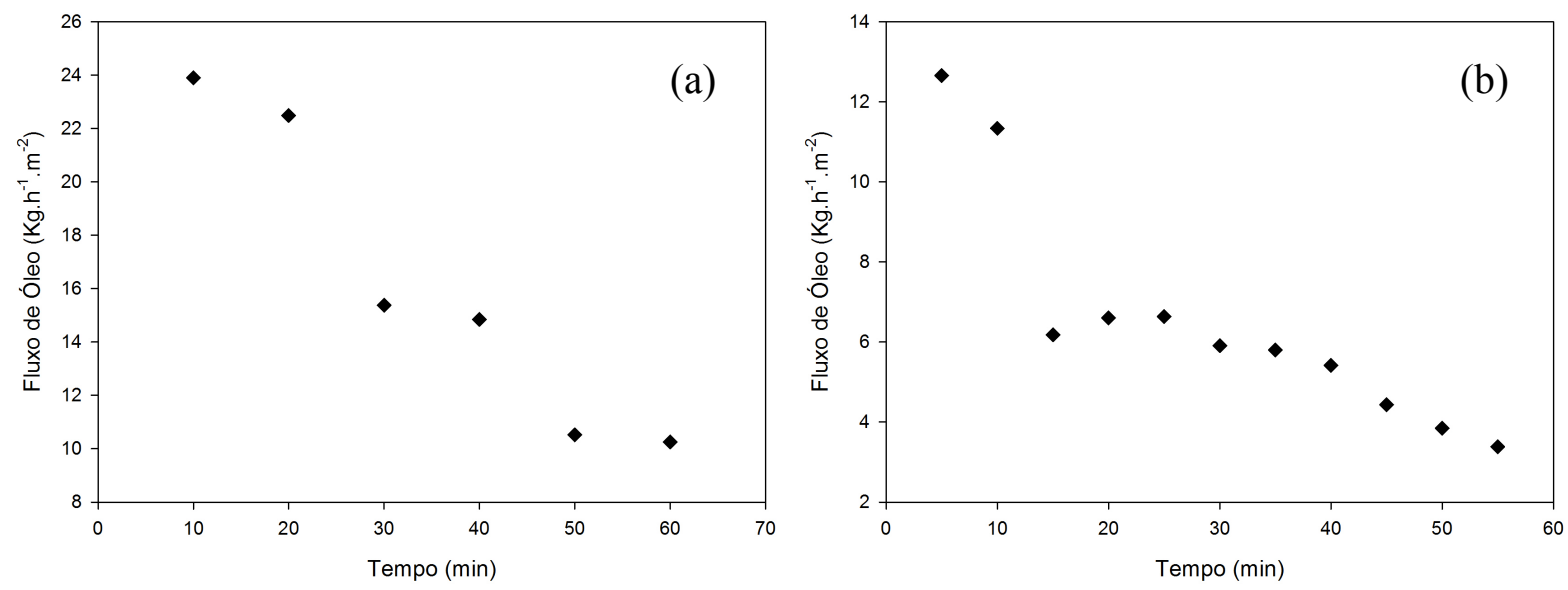

Figura 2 - Fluxo de óleo de macaúba através da membrana (a) NP030 e (b) ORAK pelo tempo

A membrana de nanofiltração NP030 mostrou maiores fluxos de óleo, com uma média em torno de $10 \mathrm{~kg} \cdot \mathrm{h}^{-1} \cdot \mathrm{m}^{-2}$, enquanto o fluxo médio para a membrana de osmose reversa ORAK foi de $6 \mathrm{~kg} \cdot \mathrm{h}^{-1} \cdot \mathrm{m}^{-2}$. Este resultado era o esperado, já que a membrana NP030 tem uma maior massa molar de corte $(\mathrm{MMCO}=400 \mathrm{Da})$, o que significa um maior diâmetro de poro. Estudos realizados por Sarrade et al. (1998) reportam baixo fluxo permeado para óleo de girassol $\left(25 \times 10^{-}\right.$ ${ }^{3} \mathrm{~kg} \cdot \mathrm{h}^{-1} \cdot \mathrm{m}^{-2}$ ) quando comparado com o fluxo permeado encontrado neste estudo com óleo de macaúba.

O índice de retenção médio de óleo pelas membranas foi de $85 \%$ e $95 \%$ para as membranas NP030 e ORAK, respectivamente. O que demonstra que não houve danos ou degradação da estrutura das membranas durante a permeação de $\mathrm{CO}_{2}$ denso, que poderiam levar a alterações nas propriedades de seletividade.

Além da retenção do óleo de macaúba, o desempenho das membranas também foi avaliado com base na capacidade de separação de ácidos graxos, uma vez que o óleo de macaúba geralmente tem um alto grau de hidrólise, e, assim, elevado teor de ácidos graxos livres. A Tabela 3 mostra os índices de retenção de cada componente do óleo de macaúba para cada uma das membranas testadas. Os índices de retenção foram determinados de acordo com a Equação 1.

Com relação aos ácidos graxos, foi observado maior retenção para o ácido palmitoléico, aproximadamente $23 \%$, com a membrana ORAK. A membrana NP030 apresentou retenções para os ácidos mirístico e palmítico, em menor proporção, cerca de $12 \%$. O fracionamento de ácidos graxos pode ser influenciado por distintos fatores, entre eles, a massa molar dos componentes, a diferença entre as membranas e, principalmente, a afinidade entre os ácidos graxos e o material de fabricação das membranas. Além disso, para ambas as membranas foram observadas retenções negativas para alguns ácidos graxos, significando neste caso a permeação total destes pelas membranas em detrimento do solvente. As diferenças de retenção observadas mostram que é possível se fracionar alguns ácidos graxos presentes na mistura . 
Tabela 3 - Índices de retenção de ácidos graxos para as membranas NP030 e ORAK na condição supercrítica

\begin{tabular}{lcc}
\hline \multicolumn{1}{c}{ Ácido Graxo } & IR \% NP030 & IR \% ORAK \\
\hline Ácido caprílico & 12,186 & 5,021 \\
Ácido cáprico & 9,222 & $-1,645$ \\
Ácido láurico & $-0,591$ & $-6,958$ \\
Ácido mirístico & 12,945 & $-6,058$ \\
Ácido palmítico & 5,902 & $-4,507$ \\
Ácido palmitoleico & 11,057 & 23,489 \\
Ácido esteárico & 8,447 & 5,519 \\
Ácido oleico & $-6,317$ & 7,007 \\
Ácido trans-13-octadecenoico & $-39,873$ & $-12,886$ \\
Ácido linoleico & $-0,687$ & $-2,957$ \\
\hline
\end{tabular}

\section{CONCLUSÕES}

Os resultados apresentados neste trabalho demonstram a possibilidade de utilização de membranas comerciais poliméricas de nanofiltração e osmose reversa para a separação de óleo de macaúba e $\mathrm{CO}_{2}$ denso. $\mathrm{O}$ estudo das membranas mostra um comportamento típico de fluxo, com redução inicial no fluxo do permeado no início do ensaio e subsequente tendência à estabilização. Ambas as membranas apresentaram boa retenção de óleo e ácidos graxos, de 85,5 \% e 95,5 \%, para NP030 e ORAK, respectivamente, indicando boa resistência ao $\mathrm{CO}_{2}$ supercrítico, podendo estas ser usadas, por exemplo, na reciclagem de $\mathrm{CO}_{2}$. Com relação ao fracionamento de ácidos graxos, as membrana de NF apresentou maior retenção, para o ácido palmitoleico, mirístico e caprílico. Já a membrana de OI foi mais seletiva ao ácido palmitoleico Observa-se que é possível a utilização deste sistema para a dessolventização de misturas de óleo de $\mathrm{CO}_{2}$ supercrítico e também para o fracionamento de ácidos graxos específicos.

\section{REFERÊNCIAS}

AKIN, O; TEMELLI, F. Effect of supercritical CO2 pressure on polymer membranes. Journal of Membrane Science. v.399- 400, p.1- 10, 2012 a.

AKIN, O.; TEMELLI, F. Performance characterization of polyamide reverse osmosis membranes upon supercritical $\mathrm{CO}_{2}$ processing. Journal of Supercritical Fluids, v.66, p.150- 156, 2012 b.

ARAKI, M.S.; COUTINHO, C.M.; GONÇALVES, L.A.G.; VIOTTO, L.A., Solvent permeability in commercial ultrafiltration polymeric membranes and evaluation of the structural and chemical stability towards hexane. Separation and Purification Technology, v. 71, p. 13 - 21, 2010.

BENEDETTI, S.; PRUDÊNCIO, E. S.; MANDARINO, J. M. G. REZZADORI, K.; PETRUS, J. C. C. Concentration of soybean isoflavones by nanofiltration and the effects of thermal treatments 
on the concentrate. Food Research International, doi:10.1016/j.foodres.2011.04.032, 2011.

CARLSON, L.H.C.; BOLZAN, A.; MACHADO, R.A.F. Separation of D-limonene from supercritical $\mathrm{CO}_{2}$ by means of membranes. Journal of Supercritical Fluids, v. 34, p. 143 - 147, 2005.

DE, B. K.; PATEL, J. D.. Effect of Different Degumming Processes and Some Nontraditional Neutralizing Agent on Refining of RBO. Journal of Oil Science, 59, (3) 121-125, 2010.

DEBON, J.; PRUDÊNCIO, E.S.; PETRUS, J.C.C. A Rheological and physico-chemical characterization of prebiotic microfiltered fermented milk. Journal of Food Engineering. v.99, p.128-135, 2010.

MARTÍNEZ, M.B.; VAN DER BRUGGEN, B.; NEGRIN, Z. R.; ALCONERO, P.L. Separation of a high-value pharmaceutical compound from waste ethanol by nanofiltration. Journal of Industrial and Engineering Chemistry. in press doi:10.1016/j.jiec.2012.02.024, 2012.

MORETTO, E.; FETT, R. Tecnologia de óleos e gorduras vegetais na indústria de alimentos. São Paulo: Livraria Varella, 1998.

MULDER, M. Basic principles of membrane technology. Center for membrane science and technology, University of Twente, Enschede, The Netherlands, p.564, 2000.

POKRYWIECKI J. C. Fracionamento do óleo essencial de manjericão de cheiro (ocimum basilicum) com membranas em meio supercrítico para concentração do linalol com reciclo do solvente no processo de extração. Tese (Doutorado em Engenharia Química - Área de Ciências de Engenharia Química). Universidade Federal de Santa Catarina, Florianópolis, 2006.

SARMENTO, L. A. V. Obtenção e separação de polifenóis de sementes de cacau por extração supercrítica associada a membranas. Tese (Doutorado em Engenharia Química). Universidade Federal de Santa Catarina, Florianópolis - SC, 2007.

SARRADE, S.; RIOS, G. M.; CARLÉS, M. Supercritical $\mathrm{CO}_{2}$ extraction coupled with nanofiltration separation. Applications to natural products. Separation and Purification Technology, v. 14, p. 19-25, 1998.

TRES, M.V.; MOHR, S.; CORAZZA, M.L.; DI LUCCIO, M.; OLIVEIRA, J.V. Separation of nbutane from soybean oil mixtures using membrane processes. Journal of Membrane Science, $\mathrm{v}$. 333, p.141-146, 2009.

Agradecimentos: Os autores agradecem ao CNPq e CAPES pelo apoio financeiro. 